\title{
Survey of Professionals' Expectations of Developmental Task Achievement of Cystic Fibrosis Self-Care in Children
}

\author{
Susana R. Patton, PhD, ${ }^{1,2 \star}$ Julie L. Graham, $\mathrm{PhD}^{2}{ }^{2}$ Douglas Holsclaw Jr., $\mathrm{MD}^{3}$ \\ and Laurie Varlotta, MD ${ }^{4}$
}

\begin{abstract}
Summary. We surveyed 174 professionals with at least 6 months of experience working with children and adolescents with cystic fibrosis (CF), to obtain estimates of ages at which $50 \%$ of children with CF may be able to perform each of 44 self-care behaviors related to the treatment of CF. Respondents were 63 physicians, 36 nurses, 24 dietitians, 18 respiratory therapists, 17 nurse practitioners, 10 social workers, and 4 psychologists, who had a mean of 11.4 years $(S D=7.5)$ of experience working with pediatric patients with CF. Mean age estimates for self-care behaviors ranged from 5.5-13.9 years. For each item, responses varied among respondents, as reflected by a mean standard deviation of 2.75 years for all behaviors. Analyses of concordance suggested a high level of agreement among respondents for the order of mastery of specific skills related to diet and enzymes use only. To date, there are no empirically defined age estimates for when children with CF may be able to perform specific self-care behaviors involved in the management of CF. This survey is the first step in generating age estimates for self-care independence in CF. Future research should conduct an objective assessment of children's CF knowledge and skill in performing these behaviors, and compare these findings to the age estimates offered in this study. Pediatr Pulmonol. 2005; 40:135-140. ๑ 2005 Wiley-Liss, Inc.
\end{abstract}

Key words: chronic illness; self-care skills; treatment independence; pediatric psychology.

\section{INTRODUCTION}

Patients with cystic fibrosis (CF) are prescribed a complex and demanding self-care regimen which they must learn to implement and adjust within their normal daily lives. For children and adolescents, it is recommended that parents and children participate actively in CF self-care behaviors to facilitate optimal adherence. ${ }^{1,2}$ Further, as children grow older, parents are encouraged to gradually transfer the primary responsibility for self-care behaviors to their children, based on their age and level of developmental readiness. ${ }^{1,3,4}$

While there is research describing children's level of independence in their $\mathrm{CF}$ regimen, ${ }^{5-8}$ no study has provided specific normative information that can guide $\mathrm{CF}$ professionals when recommending the age at which to transfer responsibility for $\mathrm{CF}$ self-care behaviors to children. In diabetes, researchers found that children follow a predictable course of diabetes self-care skill acquisition, learning rote and motoric behaviors with immediate health consequences first (e.g., complete blood glucose testing), and abstract and preventative behaviors later (e.g., anticipate and prevent hypoglycemia). ${ }^{9,10}$ Researchers also published empirically derived normative age estimates of self-care mastery for 38 skills and behaviors involved in diabetes self-care. ${ }^{11}$ These age estimates along with recommendations from the
American Diabetes Association offer physicians practical information to use in patient care. Because children with $\mathrm{CF}$ also face the challenge of mastering a complex daily treatment regimen, similar studies are needed to examine how children with $\mathrm{CF}$ may assume independence for

${ }^{1}$ Division of Child Behavioral Health, University of Michigan Health System and C.S. Mott Children's Hospital, Ann Arbor, Michigan.

${ }^{2}$ Department of Clinical and Health Psychology, MCP Hahnemann University, Philadelphia, Pennsylvania.

${ }^{3}$ Division of Pulmonary, Allergy, and Critical Care, Presbyterian Medical Center, University of Pennsylvania Health System, Philadelphia, Pennsylvania.

${ }^{4}$ Division of Pulmonology and Allergy, St. Christopher's Hospital for Children, Philadelphia, Pennsylvania.

*Correspondence to: Susana R. Patton, Ph.D., Division of Child Behavioral Health, University of Michigan Health System and C.S. Mott Children's Hospital, 1924 Taubman Center, 1500 East Medical Center Drive, Ann Arbor, MI 48109-0318. E-mail: susanap@med.umich.edu

Received 9 November 2004; Revised 10 March 2005; Accepted 14 March 2005.

DOI 10.1002/ppul.20262

Published online in Wiley InterScience

(www.interscience.wiley.com). 
CF-specific self-care behaviors, and to examine the order in which $\mathrm{CF}$ behaviors may be learned. This study surveyed CF professionals about the ages at which $50 \%$ of children with CF would be able to perform each of 44 selfcare behaviors related to a $\mathrm{CF}$ regimen.

\section{METHODS}

A total of $174 \mathrm{CF}$ professionals (physicians, nurses, dietitians, respiratory therapists, social workers, and psychologists) with at least 6 months of experience working with children with $\mathrm{CF}$ and their families participated in this study. The sample was comprised of 63 physicians, 36 nurses, 24 dietitians, 18 respiratory therapists, 17 nurse practitioners, 10 social workers, and 4 psychologists. Professionals averaged 11.4 years $(\mathrm{SD}=7.5)$ of experience working with pediatric patients with CF. They reported seeing approximately 15 $(\mathrm{SD}=16)$ patients with $\mathrm{CF}$ each week, and working in both inpatient and outpatient settings.

\section{Procedure}

Approval of the study and recruitment methods was obtained from the Institutional Review Board at MCP Hahnemann University. Six hundred fifty-one professionals were recruited to participate in this study. Participants were recruited in person at the North American Cystic Fibrosis Conference (NACFC) and at local CF centers or by mail. Of the 51 surveys distributed at the NACFC or hand-delivered to local CF centers, 30 were completed. Of the 600 surveys mailed to CF professionals in the US, 144 were returned. The overall response rate of this study was approximately $30 \%$.

Participants were asked to complete a brief employment questionnaire and a modified version of the SelfCare Independence Scale (SCIS). The SCIS is a 44-item parent-report questionnaire that was designed to obtain information regarding children's level of self-care independence in CF-specific self-care tasks. ${ }^{8}$ For the current study, the directions of the SCIS were modified, and professionals were asked to provide estimates of the ages at which at least $50 \%$ of children with CF may be expected to have mastered each of 44 behaviors. Similar to previous studies, mastery of a behavior was defined as the stage when "a child is independent in initiating/performing the skill and/or performing the behavior without receiving prompting from an adult or

\footnotetext{
ABBREVIATIONS

CF Cystic fibrosis

MANOVA Multivariate analysis of variance

NACFC North American Cystic Fibrosis Conference
}

requiring information about how to perform the behavior correctly." $, 9,11,12$

\section{Statistics}

Preliminary analyses were conducted to examine any relationship between respondent characteristics and item responses for the SCIS. For these analyses, a Bonferroni correction was applied to control for family-wise error. Findings of a series of Pearson product-moment correlations revealed no relationships between age estimates on the survey and respondents' average patient load per week ( $\mathrm{r}$ range $=0.001-0.11)$ or years of experience in $\mathrm{CF}(\mathrm{r}$ range $=0.004-0.22$ ). Likewise, results of two multivariate analysis of variance (MANOVA) tests revealed no differences in age estimates due to the method of data collection (e.g., mailed vs. in person, $\mathrm{F}(1,112)=1.47$, $P=0.09$ ) or respondent's professional role (e.g., physician, nurse, dietitian, respiratory therapist, social worker, or psychologist; $\mathrm{F}(4,110)=1.23, P=0.08)$. To examine normative age estimates, behaviors of the SCIS were analyzed according to means, standard deviations, medians, and percentages. Mean standard deviations were calculated to assess variability of respondents' responses on individual SCIS behaviors. Finally, to assess the rate of agreement among respondents for order of skill mastery, individual behaviors were sorted according to median age estimates within each of six treatment domains (e.g., aerosol, lung clearance, diet, enzyme, medications, and general care). The order of skill mastery within these domains was examined according to Kendall's coefficient of concordance.

\section{RESULTS}

Table 1 presents, for all 44 self-care skills, mean ages and ages at which $25 \%, 50 \%$, and $75 \%$ of professionals estimated mastery for children with CF. Overall, mean independence ages provided by professionals ranged from 5.5 years ("know the name of the pulmonologist") to 13.9 years ("remember the time of each appointment"), suggesting that $50 \%$ of children with $\mathrm{CF}$ may have mastery over basic CF self-care behaviors by age 14 years. Responses to each of the 44 self-care items demonstrated some variability, with a mean standard deviation of 2.75 years for all items (individual-item standard deviations ranged from 2-4 years). In addition, there was variability when items were grouped according to treatment domain (e.g., aerosol, lung clearance, diet, enzyme, medications, and general). These analyses revealed mean standard deviations ranging from 2.34 years among aerosol items to 2.90 years among diet items. To assess the rate of agreement among professionals for the order of skill mastery within treatment domains, a series of Kendall's coefficients of concordance was calculated, 
TABLE 1-Median, Percentile, and Mean Scores of Age Estimates of Skill Mastery Reported by Professionals

\begin{tabular}{|c|c|c|c|c|}
\hline Self-care behaviors & $25 \%$ & $50 \%$ & $75 \%$ & Mean $\pm \mathrm{SD}$ \\
\hline \multicolumn{5}{|l|}{ Aerosol behaviors } \\
\hline Use an aerosol? & 6.0 & 7.0 & 9.0 & $7.2 \pm 2.3$ \\
\hline Know when to perform each aerosol treatment? & 8.0 & 9.0 & 10.0 & $9.2 \pm 2.2$ \\
\hline Name prescribed aerosols? & 8.0 & 10.0 & 10.5 & $9.6 \pm 2.3$ \\
\hline \multicolumn{5}{|l|}{ Lung clearance behaviors } \\
\hline Know how many times each day to perform CPT? & 6.0 & 8.0 & 9.0 & $7.9 \pm 2.5$ \\
\hline Explain why to perform CPT? & 7.0 & 8.0 & 10.0 & $8.7 \pm 2.7$ \\
\hline Enlist the help of another to perform CPT? & 7.0 & 8.0 & 12.0 & $9.2 \pm 3.9$ \\
\hline Use a therapy vest? & 7.0 & 8.0 & 10.0 & $8.5 \pm 2.8$ \\
\hline Know when to use the therapy vest? & 7.0 & 8.0 & 10.0 & $8.9 \pm 2.7$ \\
\hline Know how many times each day to use the therapy vest? & 7.0 & 8.0 & 10.0 & $8.6 \pm 2.5$ \\
\hline Know how many times each day to use the Flutter? & 7.0 & 9.0 & 10.0 & $9.1 \pm 2.5$ \\
\hline Know how long to perform each treatment? & 8.0 & 10.0 & 11.3 & $9.6 \pm 2.7$ \\
\hline Name prescribed medications to be taken before CPT? & 8.0 & 10.0 & 12.0 & $9.9 \pm 2.6$ \\
\hline \multicolumn{5}{|l|}{ Diet behaviors } \\
\hline Remember the number of meals and snacks to eat in a day? & 7.0 & 8.0 & 10.0 & $8.6 \pm 2.7$ \\
\hline Know to eat high-fat/high-calorie foods? & 7.0 & 9.0 & 10.0 & $9.0 \pm 2.5$ \\
\hline Name high-calorie nutritional supplements? & 7.0 & 9.0 & 10.0 & $9.1 \pm 2.7$ \\
\hline Pick out high-calorie foods? & 8.0 & 10.0 & 12.0 & $10.0 \pm 2.6$ \\
\hline Monitor changes in weight? & 8.0 & 10.5 & 12.0 & $10.6 \pm 2.8$ \\
\hline Explain how the body uses food as energy? & 8.0 & 11.0 & 12.0 & $9.7 \pm 2.9$ \\
\hline Know when to take high-calorie nutritional supplements? & 8.0 & 10.0 & 12.0 & $10.7 \pm 2.9$ \\
\hline Know how many nutritional supplements to take each day? & 8.0 & 10.0 & 12.0 & $10.2 \pm 2.8$ \\
\hline Eat recommended amounts of foods? & 8.0 & 10.0 & 13.0 & $11.0 \pm 3.5$ \\
\hline Make changes in their diet to adjust for weight loss? & 11.0 & 12.0 & 14.0 & $12.3 \pm 2.8$ \\
\hline \multicolumn{5}{|l|}{ Enzyme behaviors } \\
\hline Take enzymes in capsule form? & 5.0 & 6.0 & 8.0 & $6.3 \pm 2.4$ \\
\hline Know when to take enzymes? & 5.0 & 7.0 & 8.0 & $7.0 \pm 2.7$ \\
\hline Know how many enzymes to take with meals and snacks? & 5.0 & 7.5 & 9.0 & $7.5 \pm 2.5$ \\
\hline Explain what enzymes do in the body? & 7.0 & 8.3 & 10.5 & $9.0 \pm 2.6$ \\
\hline Learn the brand name(s) of enzymes prescribed? & 7.0 & 9.0 & 10.0 & $9.0 \pm 2.7$ \\
\hline Adjust the number of enzymes based on foods eaten? & 10.0 & 12.0 & 13.5 & $11.7 \pm 2.4$ \\
\hline \multicolumn{5}{|l|}{ Medication and vitamin behaviors: } \\
\hline Swallow vitamins with water? & 5.0 & 6.0 & 8.0 & $6.9 \pm 2.3$ \\
\hline Use nasal sprays? & 6.0 & 8.0 & 10.0 & $7.9 \pm 2.1$ \\
\hline Remember to take vitamins? & 6.3 & 8.5 & 10.0 & $8.9 \pm 3.1$ \\
\hline Name the vitamins prescribed? & 7.0 & 8.0 & 10.0 & $8.5 \pm 2.4$ \\
\hline Remember the dose of each vitamin? & 7.0 & 9.0 & 11.0 & $9.4 \pm 3.1$ \\
\hline Know when to use nasal sprays? & 8.0 & 10.0 & 11.0 & $9.6 \pm 2.2$ \\
\hline Name the nasal sprays prescribed? & 8.0 & 10.0 & 11.5 & $9.7 \pm 2.1$ \\
\hline Explain what antibiotics do in the body? & 9.0 & 12.0 & 13.0 & $11.4 \pm 3.2$ \\
\hline Know when to take antibiotics? & 10.0 & 12.0 & 12.0 & $11.4 \pm 2.7$ \\
\hline Learn the types of antibiotics prescribed? & 10.0 & 12.0 & 13.0 & $11.4 \pm 2.7$ \\
\hline Name what antibiotics are prescribed? & 10.0 & 12.0 & 13.0 & $11.8 \pm 2.7$ \\
\hline Take antibiotics as prescribed? & 10.0 & 12.0 & 14.0 & $12.0 \pm 3.1$ \\
\hline \multicolumn{5}{|l|}{ General knowledge } \\
\hline Name his or her pulmonologist? & 4.0 & 5.0 & 6.0 & $5.5 \pm 2.1$ \\
\hline Talk to others about cystic fibrosis? & 8.0 & 10.0 & 12.0 & $9.9 \pm 2.7$ \\
\hline Recognize the symptoms of an infection and tell someone about it? & 8.0 & 10.0 & 12.0 & $10.2 \pm 2.6$ \\
\hline Remember the time of each appointment? & 12.0 & 14.0 & 16.0 & $13.9 \pm 2.7$ \\
\hline
\end{tabular}

using the median age estimates for each behavior within each treatment domain. These analyses revealed close agreement for the order of skill mastery for behaviors in the diet $(\mathrm{W}=0.60, P=0.001)$ and enzyme $(\mathrm{W}=0.89$, $P=0.001)$ domains. However, the rates of agreement for the other four treatment domains were not significant, suggesting discordance among professionals as to the order of skill mastery within these domains.

\section{DISCUSSION}

Achieving personal autonomy is a lifelong process. Research has documented that most children and adolescents follow a predictable course with regard to cognitive and social development. ${ }^{13}$ Similarly, research indicates that children's achievement of specific self-care tasks closely follows their level of cognitive and social 
development. That is, children typically first learn skills that are routine, concrete, and mechanistic. Later, as they become more cognitively advanced, they gain insight into behaviors, learning the purpose for behaviors, how to manipulate behaviors, and how to evaluate the consequences of behaviors. $^{13}$

This study surveyed 174 professionals with at least 6 months of experience working with children with $\mathrm{CF}$ about the ages when they would expect $50 \%$ of children with $\mathrm{CF}$ to have learned 44 self-care behaviors related to a $\mathrm{CF}$ treatment regimen. Findings revealed item mean age estimates that ranged from 5.5-13.9 years. A visual survey of the data suggested that behaviors judged to require mechanical skills and rote learning (e.g., swallowing capsules, remembering the name of the pulmonologist) were expected to be acquired at an earlier age than those skills requiring judgment, decision-making, or task adjustment (e.g., making dietary changes, taking antibiotics as prescribed). This finding is consistent with the developmental literature and the predicted pace at which children grow and develop thinking and problem-solving skills. ${ }^{9,11,13}$ Moreover, analyses of concordance revealed agreement across professionals for the order of behaviors in the dietary and enzyme domains. This finding has important implications for the development and evaluation of educational programs for $\mathrm{CF}$, especially with regard to dietary recommendations, which are the foundation of treatment for the majority of children and adolescents with $\mathrm{CF} .{ }^{14}$

A more refined analysis of the data revealed some variability among predicted age estimates for each of the 44 items. An examination of the mean standard deviations of each treatment domain revealed the greatest variability among dietary behaviors and the least variability among behaviors related to aerosol use. The SCIS lists 10 behaviors within the dietary treatment domain. It is expected that these behaviors vary considerably with regard to the underlying skills needed to achieve mastery. For example, the behavior "know to eat high-calorie/fat foods" is a rote skill that likely taps children's memory abilities, but involves little problem-solving. In contrast, the behavior "eat recommended amounts of foods" is a skill that may tap children's memory, organization, and conceptual reasoning abilities. That is, to be successful with this task, children need to be able to plan meals, remember what they have eaten over the course of the day, and make adjustments to meals/serving sizes to maintain an appropriate calorie intake. Interestingly, in a smaller study, parents reported a mean standard deviation of 2.85 years for age estimates of dietary items on the SCIS, which is consistent with the current findings among professionals. ${ }^{15}$ Parents and professionals did diverge when examining individual items. In the current study, the item "eat recommended amounts of foods" had a mean standard deviation of 3.54 years, which was the largest of the items in the dietary domain. However, in the parent study, the item "remember the number of meals/snacks" had the widest variability $(\mathrm{SD}=3.37)$ among dietary items. ${ }^{15}$ It is expected that these items are highly related with regard to the knowledge and skills needed for mastery. Therefore, it is possible that the disparity may be due to differences in how parents and professionals conceptualize dietary self-care, and whether the emphasis need be on total energy intake per day or regular energy intake across the day.

This is the first study to survey professionals about their expectations for the ages at which children with $\mathrm{CF}$ might be able to perform specific self-care tasks. Previous research investigating parents' reports of children's mastery levels found older age estimates for dietary behaviors and overall lower expectations of independence for self-care behaviors for children and adolescents. ${ }^{5,15}$ While it is possible that those parents offered overly conservative estimates of their children's self-care abilities, it is also possible that the survey directions used in the current study influenced the age estimates provided by professionals. In the current study, professionals were asked to consider the abilities of children with CF falling at the median of age estimates of skill mastery. These directions required professionals to make generalizations across patients and to ignore individual differences which could impact predicted age estimates. It is possible that professionals would have offered older age estimates if they had been asked to consider a single patient in their clinic or to consider the abilities of all children with CF.

While no study has asked professionals to provide age estimates of self-care autonomy in CF, two studies asked professionals to provide age estimates of when it is best to begin to transition adolescents with $\mathrm{CF}$ to an adult care center. ${ }^{3,4}$ In those studies, physicians, nurses, social workers, dietitians, respiratory therapists, and other health professionals were asked to give the age at which they typically introduce the transition process to patients. In both studies, professionals reported that they typically introduce patients to the transition process at approximately 16 years of age. Further, physicians reported a mean age of $18.5 \pm 1.8$ years for when they typically transition adolescents to adult centers. ${ }^{3}$ While the survey instructions for these studies did not specify that respondents should consider the median age for transition, it is interesting to note that these ages are higher than the age estimates for $\mathrm{CF}$ self-care reported in the current study. In the literature it is unclear why professionals report age estimates for transition in middle to late adolescence. ${ }^{4}$ It is possible that professionals prefer to delay the transition of pediatric patients into later adolescence so that these transitions coincide with other developmental milestones of this age range, such as learning to drive or moving away from home. Alternatively, 
professionals may choose to delay transition so that they have more time to assess that their patients are adequately equipped for transition with regard to disease knowledge, self-care skills, and treatment motivation. These disparate age estimates present an interesting research question which should be investigated in order to better understand the transition process and to design programs to enhance patients' transition to adult centers.

\section{Limitations}

This survey provides preliminary normative age estimates for 44 self-care behaviors included in a typical $\mathrm{CF}$ treatment regimen. These data were collected empirically and were based on the collected experience of 174 professionals who work with children with CF. The process of preparing a child to assume more responsibility for his/her self-care is difficult and dependent on the cognitive and emotional readiness of the child, as well as his/her age. Because it is difficult to assess a child's cognitive and emotional readiness, age is a common proxy measure of maturity and ability. However, age is an imperfect measure of these factors. Therefore, additional research is needed to assess the accuracy of current age estimates via an objective assessment of children's CF knowledge and self-care skills. The directions of the SCIS prompted professionals to make generalizations about expected mastery ages, which could have been influenced by an availability bias if professionals relied too heavily on recent or particularly salient interactions with very competent or incompetent patients. This could have introduced error, with some professionals overestimating and others underestimating skill-mastery ages. Future research in this area should consider modifying the survey directions to minimize the potential for error caused by generalizations. One suggestion, which would not completely remove error but might help to minimize its effect, is to ask professionals to record separately the skill mastery of their most competent patients and their patients who require more family assistance, and to present these age estimates as the upper and lower limits of skill mastery. While this study had a low response rate, to date, these data represent the most scientifically obtained estimates of professionals' expectations for CF self-care achievement, and these data fill an important gap in transition research in CF. Similar survey studies in CF have enjoyed a higher response rate or total sample of respondents. ${ }^{3,4}$ For one of these studies, the researchers found an internet-based format to be very effective in recruiting professionals. ${ }^{4}$ Presently, there are several websites that provide user-friendly formats for collecting survey data via the internet. Future research should consider using an internet-based survey in order to attract a larger sample of respondents.
As children with CF grow up, it is important that they assume more responsibility for their CF self-care, so that they may be better prepared for the developmental milestones of childhood and adolescence and the transition to adult CF centers. In the absence of guidelines to help direct the appropriate transfer of self-care responsibilities from parents to children, it is difficult to identify patients who are developing at a predicted pace vs. patients who may have an inappropriate level of self-care independence. ${ }^{8}$ This survey provides preliminary normative age estimates of self-care autonomy for children and adolescents with $\mathrm{CF}$. It is expected that these age estimates may be useful guidelines for physicians and parents to use in patient care.

\section{ACKNOWLEDGMENTS}

We thank James Herbert, Ph.D., Michael Lowe, Ph.D., Patrick McGuffin, Ph.D. (MCP Hahnemann University), and Scott W. Powers, Ph.D. (Cincinnati Children's Hospital Medical Center), for their technical assistance in the design of this project and the presentation of data. We also thank the community of physicians, nurses, respiratory therapists, dietitians, social workers, and psychologists recruited to participate in this project.

\section{REFERENCES}

1. American Academy of Pediatrics Committee on Children with Disabilities and Committee on Adolescence. Transition of care provided for adolescents with special health care needs. Pediatrics 1996;98:1203-1206.

2. Sawyer SM, Blair S, Bowes G. Chronic illness in adolescents: transfer or transition to adult services? J Paediatr Child Health 1997;33:88-90.

3. Flume PA, Anderson DL, Hardy KK, Gray S. Transition programs in cystic fibrosis centers: perceptions of pediatric and adult program directors. Pediatr Pulmonol 2001;31:443-450.

4. Flume PA, Taylor LA, Anderson DL, Gray S, Turner D. Transition programs in cystic fibrosis centers: perceptions of team members. Pediatr Pulmonol 2004;37:4-7.

5. Drotar D, Ievers C. Age differences in parent and child responsibilities for management of cystic fibrosis and insulindependent diabetes mellitus. J Dev Behav Pediatr 1994;15:265272.

6. Gudas LJ, Koocher GP, Wypij D. Perceptions of medical compliance in children and adolescents with cystic fibrosis. J Dev Behav Pediatr 1991;12:236-242.

7. Cappelli M, MacDonald NE, McGrath PJ. Assessment of readiness to transfer to adult care for adolescents with cystic fibrosis. Child Health Care 1989;18:218-224.

8. Patton SR, Graham JL, Varlotta L, Holsclaw D Jr. Measuring selfcare independence in children with cystic fibrosis: the SelfCare Independence Scale (SCIS). Pediatr Pulmonol 2003;36: $123-130$

9. Wysocki T, Meinhold PA, Abrams KC, Barnard MU, Clarke WL, Bellando BJ, Bourgeois MJ. Parental and professional estimates of self-care independence of children and adolescents with IDDM. Diabetes Care 1992;15:43-52. 
10. Dashiff C, Bartolucci A. Autonomy development in adolescents with insulin dependent diabetes mellitus. J Pediatr Nurs 2002;17: 96-106.

11. Wysocki T, Meinhold P, Cox DJ, Clarke WL. Survey of diabetes professionals regarding developmental changes in diabetes selfcare. Diabetes Care 1990;13:65-68.

12. Wysocki T, Meinhold PM, Taylor A, Hough BS, Barnard MU, Clarke WL, Bellando BJ, Bourgeois MJ. Psychometric properties and normative data for the parent version of the diabetes independence survey. Diabetes Educ 1996;22:587-591.
13. Thomas R. Developmental tasks. In: Thomas RM, editor. The encyclopedia of human development and education: theory, research, and studies. New York: Pergamon Press; 1989. p 54-60.

14. Borowitz D, Baker RD, Stallings V. Consensus report on nutrition for pediatric patients with cystic fibrosis. J Pediatr Gastroenterol Nutr 2002;35:246-259.

15. Patton SR, Graham JL, Varlotta L, Holsclaw D Jr, Waddell C, Fakaros M. Parental and professional age-estimates of dietary adherence in children with cystic fibrosis. Pediatr Pulmonol [Suppl] 2000;30:548. 